\title{
La importancia de la educación ambiental ante la problemática actual
}

\author{
Róger Martínez, Castillo \\ Universidad Nacional de Costa Rica y Universidad de Costa Rica \\ Costa Rica
}

Recibido 15 de febrero de 2010 • Aceptado 17 de marzo de 2010

\begin{abstract}
Resumen. El sistema de desarrollo dominante, con sus políticas neoliberales y enfoques mercadocéntricos contribuye a agravar los problemas socio-ambientales, bajo un contexto de la globalización del mercado. Por eso, la preocupación por el manejo sustentable del ambiente, hace imperiosa la necesidad de estructurar una educación ambiental que forme e informe acerca de esta problemática. En este sentido, la educación ambiental viene a constituir el proceso educativo que se ocupa de la relación del ser humano con su ambiente (natural y artificial) y consigo mismo, así como las consecuencias de esta relación. De esta manera, la educación ambiental debe constituir un proceso integral, que juega su papel en todo el entramado de la enseñanza y el aprendizaje. Para ello, es necesario establecer un proceso educativo que cuestione la relación de cualquier tema o actividad del ser humano, dentro de un análisis de la importancia o incidencia en la vida social y ambiental, como es la parte pedagógica y su esencia política.
\end{abstract}

Palabras clave. Educación, ambiente, sociedad, globalización y sustentabilidad.

Abstract. The dominant development system with its neoliberal policies and market-centered approaches contribute to aggravate the socio-environmental problems, under the context of the globalization of the market. For that reason, the concern about the sustainable development of the environment makes highly necessary the structuring of an environmental education that forms and informs about this problem.

As a result, environmental education constitutes the educational process that deals with the relation between the human being and his/her environment (natural and man-made) and with itself, as well as the effects caused by this relation. In this way, environmental education must constitute an integral process, which plays its role throughout the whole teaching and learning intertwinement. For this reason it is necessary to establish an educational process that examines the relation of any topic or human being activity, within an analysis of the importance or incidence on social life, as it is the pedagogical part and its political essence.

Key words. Education, environment, society, globalization and sustainability.

\footnotetext{
${ }^{1}$ Doctorado Agroecología (2003), Universidad de Córdoba, España. Maestría en Agroecología (2003), Universidad Internacional de Andalucía, España. Licenciatura en Educación Ambiental (2000), Universidad Nacional, Costa Rica. Máster en Educación Ambiental (1998), IIE, Málaga, España. Maestría en Historia (Énfasis en Arqueología) (1984), Universidad de Vorónezh, Rusia. Profesor Catedrático Universidad de Costa Rica y Catedrático Universidad Nacional de Costa Rica. Ha participado en más de noventa congresos, seminarios nacionales e internacionales, ha sido invitado a nueve países; ha publicado dos libros y más de 130 ponencias, ensayos y artículos en diversas revistas nacionales e internacionales. Correo electrónico: rmartine@una.ac.cr
}

La Revista Electrónic@ Educare está indizada en el Catálogo LATINDEX.

Artículo protegido por la licencia Creative Commons. 


\section{Introducción}

A escala planetaria, el ser humano está poniendo en peligro su vida a causa del modo en que se desarrollan sus relaciones con el medio ambiente.

La sociedad es un mundo de grandes desequilibrios e injusticias, en la que riqueza y lujo (de minorías) se codean con la pobreza y miseria más cruda (de mayorías), el proceso de apropiación, producción y consumo, y el crecimiento explosivo poblacional agravan la situación de la propia biosfera que está siendo degradada.

La globalización neoliberal estimula la polarización entre países y sectores ricos y pobres, y acentúa, aún más, la brecha (económica, tecnológica, comercial, productiva) en los ámbitos nacional e internacional. La complejidad que la globalización ha imprimido a las relaciones entre los países y las personas, entre el Norte rico y el Sur empobrecido, y la actual crisis ecológica hace urgente la tarea de explorar nuevas alternativas.

La actual crisis ecológica -provocada por el impacto de las actividades humanas y el modelo de vida occidental- se unen a otros síntomas desestabilizadores, como son las fracturas económicas - con fuertes desigualdades mundiales en las condiciones de vida de sus habitantes-, sociales -expresadas en exclusiones de distinto signo- y culturales -xenofobia vinculada a la idea dominante de unas culturas sobre otras-. Aun en los espacios del planeta donde no hay conflictos armados, aparecen múltiples indicadores de un cierto tipo de guerra, una guerra del ser humano contra su entorno y contra sí mismo (Hernández, Ferriz, Herrero, González, Morán, Brasero et.al., 2010).

Por eso, las formas de vivir, pensar, producir, valorar, utilizar, contaminar son el reflejo histórico de un determinado nivel de desarrollo socio-histórico, con dinámica propia, el cual es aprendido, compartido, transmitido socio-culturalmente, según las necesidades e intereses del ser humano abarca todas las acciones humanas: modos de pensar, sistemas de valores y símbolos, costumbres, religión, instituciones, organizaciones, economía, comercio e intercambio, producción, educación, legislación, entre muchos otros aspectos de la acción humana, por ende, de la creación de cultura. (Martínez, 2007b).

Los problemas ambientales se presentan tanto en el nivel nacional como en el internacional, sin que se puedan resolver, a causa de que los intereses de pocos, están antepuestos a las necesidades de todos. Además, éstos se manifiestan de manera trascendente y general, como por ejemplo: el deterioro de la capa de ozono, el efecto de invernadero, el cambio climático, sin que sea tangible el problema para la población y sin la debida participación activa y decisiva de la comunidad ante sus problemas.

El actual modelo de desarrollo dominante da prioridad a una economía insustentable e injusta socialmente, que nos lleva a una crisis de vida en la ecoesfera. El deterioro socio-ambiental debe replantear la vinculación de la sociedad con su entorno natural, mediante un desarrollo sustentable, y reconocer la existencia de límites al desarrollo social, al crecimiento económico (productivo) y a la explotación (abuso) de los ecosistemas, dado el estado actual de la tecnología, la organización social y la capacidad de la biosfera para absorber los efectos de las actividades humanas.

Por tanto, el deterioro ambiental se agudiza debido a la voracidad del sector privado desregularizado (nacional y transnacional) y público (Ministerios, instituciones y gobierno) por los recursos naturales como el agua, el suelo, la tierra, el bosque, los minerales, las bellezas escénicas (Martínez, 2007a). El sector público se muestra incapaz y, todavía, en general, apoya al sector privado voraz. Se acentúan los problemas ambientales y el gobierno no cuenta con mecanismos eficientes de control, ordenamiento físico, jurídico y operativo para lograr un manejo sustentable de estos recursos. 
Hace décadas, el modelo de desarrollo actual manifiesta síntomas de degradación y ha alcanzado tal magnitud, que escapa del control humano; así, por primera vez en la historia de la humanidad, se pone en peligro la vida en el planeta, tal como se presenta en la Tabla 1.

Tabla 1

Rasgos globales de la crisis social y ambiental

$\sqrt{ }$ El estilo de vida humano es muy destructivo de las relaciones sociedad-biosfera.

$\sqrt{ }$ La idea aristotélica de lo humano y sus actividades separadas de lo natural, es decir, el concepto antropocéntrico predominante en la relación especie humana-naturaleza.

$\sqrt{ }$ El enfoque mercado-céntrico, su forma de dar valor a las cosas y su énfasis en la ganancia.

$\sqrt{ }$ Crecimiento continuo e ilimitado, basado en una economía de libre mercado (desregularizada), que abusa de los ecosistemas y seres humanos.

$\sqrt{ }$ Estilo de vida irracional (superproducción, sobreconsumo y derroche) para pocas(os) y relaciones sociales injustas para las mayorías (limitación productiva, carencias en el consumo, abstención).

$\sqrt{ }$ Individualidad e inviolabilidad de los derechos de propiedad privada, en detrimento de la colectividad social y lo ambiental.

$\sqrt{ }$ Inconsciencia de la unidad del ecosistema planetario que niega la interdependencia ecológica y económica en el mundo.

$\sqrt{ }$ Deterioro de las fuentes de energías fósiles, no renovables cuyos impactos económicos obliga no sólo a ahorrarlos, sino a la búsqueda de alternativas limpias y renovables.

$\sqrt{ }$ Desarrollo tecnológico y social abre una brecha entre dos sectores: el mundo desarrollado (rico) y el mundo en desarrollo (pobre), mediante relaciones asimétricas, intercambios desiguales e injustos en lo económico y lo tecnológico, en las que el desequilibrio repercute en el ambiente y la sociedad.

Nota. Recopilación propia.

Los problemas ambientales ya no aparecen como independientes unos de otros, sino que constituyen elementos que se relacionan entre sí y configuran una realidad diferente a la simple acumulación de todos ellos. No podemos limitarnos a percibir esta crisis como un conflicto entre determinados planteamientos sobre el mundo y sobre la vida, de manera inadecuada (Beck, 2004).

Una política educativa integral es difícil de aplicar porque necesita del poder político (intereses internos y externos) para ponerse en práctica, a causa de que esta crisis ha despertado la necesidad de superar viejos paradigmas y mitos. Aquí, la educación tiene un importante papel que jugar, en la promoción de un aprendizaje innovador caracterizado por la anticipación y la participación que permita no sólo comprender, sino, también, implicarse en aquello que queremos entender (Fien, Scott y Tilbury, 1999).

\section{Educación y sociedad}

Por educación se entiende un proceso de desarrollo socio-cultural continuo de las capacidades que las personas en sociedad deben generar y que se realiza tanto dentro como fuera de su entorno, a lo largo de toda la vida. La educación implica impulsar las destrezas y las estructuras cognitivas, que permiten que los estímulos sensoriales y la percepción del mundorealidad se conviertan de información significativa, en conocimientos de su construcción y reconstrucción, así como en valores, costumbres, que determinan nuestros comportamientos o formas de actuar (Álvarez, 2003). 
La educación es necesaria para todo ser humano, pues bien orientada e integral puede servirle para interpretar su realidad, ya que relaciona sus distintos componentes y conforma un universo de posibilidades, aprende y sustenta su ubicación en la sociedad, en general, y de la vida, en particular.

El desarrollo socio-económico juega un papel importante en este proceso, en el que la educación, como influencia socio-cultural, es un instrumento de transmisión de conocimientos, experiencias e identidad (Martínez, 1998). Así, la educación reproduce valores y técnicas sociales, que contribuyen a su metabolismo, por lo que, la educación es la mejor vía para organizar la transformación social y ambiental.

La educación contribuye a una conciencia crítica e integral de nuestra situación en el planeta. También, es un agente importante en la transición a una nueva fase ecológica de la humanidad. Pretende comprender su relación en la biosfera humanizada, al formar personas capaces de interpretar y transformar el mundo, y de dar importancia a los derechos de todos los seres vivos (incluyendo humanos) y la naturaleza, para contribuir a plantear políticas y culturas basadas en necesidades a corto plazo (Freire, 1995).

Educar como proceso permite la construcción, la reconstrucción y la reflexión de conocimientos, conductas de valores y el desarrollo de las capacidades individuales y colectivas. La educación como mecanismo de adaptación cultural del ser humano al ambiente, se ha mostrado poco crítica con respecto a las actitudes y comportamientos ambientales. Es necesario redimensionarla, mediante el impulso de una acción formativa dirigida al cambio actitudinal y la modificación de comportamientos colectivos. El crecimiento moral se facilita cuando se aprovechan las situaciones de conflicto o lo que afecta a las personas, y que las obliga a tomar partido. Trata de que el individuo cuestione sus ideas y conductas, que critique sus creencias-valores y los de su grupo social (Caduto, 1992; Puig Rovira, 1992).

\section{Educación ambiental: definiciones}

La UNESCO (1980), en una magna Conferencia sobre Educación Ambiental, plantea los objetivos de la educación ambiental en los siguientes términos:

a. Comprender la naturaleza compleja del ambiente resultante de la interacción de sus aspectos biológicos, físicos, sociales y culturales.

b. Percibir la importancia del ambiente en las actividades de desarrollo económico, social y cultural.

c. Mostrar las interdependencias económicas, políticas y ecológicas del mundo moderno en el que las decisiones y los comportamientos de todos los países pueden tener consecuencias de alcance internacional.

d. Comprender la relación entre los factores físicos, biológicos y socioeconómicos del ambiente, así como su evolución y su modificación en el tiempo.

Aunque es difícil encuadrar la educación ambiental, se puede partir de la propuesta del Congreso de Moscú, de 1987 (citado por Labrador y del Valle, 1995):

La educación ambiental es un proceso permanente en el cual los individuos y las comunidades adquieren conciencia de su ambiente, aprenden los conocimientos, los valores, las destrezas, la experiencia y, también, la determinación que les capacite para actuar, individual y colectivamente, en la resolución de los problemas ambientales presentes y futuros. 
Pero, el concepto de educación ambiental no es estático, evoluciona de forma paralela a como lo hace la idea de medio ambiente y la percepción que se tiene. Hoy, las dimensiones socioculturales, políticas y económicas son básicas para entender las relaciones que la humanidad establece con su medio (Bedoy, 2000).

El objetivo general de la educación ambiental es que ésta como proceso educativo se encuentra ampliamente ligada a las características políticas y económicas de las naciones. En este sentido, la educación ambiental nace haciendo de la naturaleza un bien universal y no manejable por los intereses particulares de nadie.

La educación ambiental debe ir dirigida a toda la humanidad debido a que es un problema que incumbe a todos (Wood y Walton, 1990).

$\mathrm{Su}$ importancia consiste en lograr que los individuos y las colectividades comprendan la naturaleza compleja del ambiente natural y del creado por el ser humano, que sea resultado de la interacción de sus aspectos biológicos, físicos, sociales, económicos y culturales; y adquieran los conocimientos, los valores, los comportamientos y las habilidades prácticas para participar responsable y eficazmente en la prevención y la solución de los problemas ambientales, y en la gestión relacionada con la calidad ambiental (Caduto, 1992).

La educación ambiental puede y debe ser un factor estratégico que incida en el modelo de desarrollo establecido para reorientarlo hacia la sustentabilidad y la equidad. "Para contribuir con eficacia a mejorar el ambiente, la acción de la educación debe vincularse con la legislación, las políticas, las medidas de control y las decisiones que los gobiernos adopten, en relación con el ambiente humano" (UNESCO, 2004, s. p.).

Los objetivos de la educación ambiental, se deben basar en:

a) Considerar al ambiente, en forma integral, o sea, no sólo los aspectos naturales, sino los tecnológicos, sociales, económicos, políticos, morales, culturales, históricos y estéticos.

b) Asumir un enfoque transdisciplinario para el tratamiento ambiental, inspirado en cada disciplina, para posibilitar una perspectiva equilibrada.

c) Tratar la temática ambiental desde lo particular a lo general tiene como finalidad que los estudiantes se formen una idea de las condiciones ambientales de otras áreas, que identifiquen las condiciones que prevalecen en las distintas regiones geográficas y políticas, además de que reflexionen sobre las dimensiones mundiales del problema ambiental para que los sujetos sociales se involucren en los diferentes niveles de participación y responsabilidad.

d) Promover el conocimiento, la habilidad para solucionar problemas, la clasificación de valores, la investigación y la evaluación de situaciones, para aprender sobre la propia comunidad.

e) Capacitar a los estudiantes para que desempeñen un papel en la planificación de sus experiencias de aprendizaje y dejarles tomar decisiones y aceptar sus consecuencias.

La educación ambiental abarca algo más que el estudio de relaciones pedagógicas y ecológicas; trata de las responsabilidades políticas que debe tener el sistema educativo formal, de preparar a los educandos para que sean capaces de generar los cambios necesarios que aseguren un desarrollo sustentable, así como estimular conciencia para la solución de los problemas socio-ambientales actuales (Caride, 2000).

La educación ambiental debe generar cambios en la calidad de vida, en la conducta personal $\mathrm{y}$ en las relaciones humanas, que lleven a la solidaridad y el cuido hacia todas las formas de vida y el planeta (Febres y Florián, 2002). 
Al respecto, la educación ambiental pretende:

- Favorecer el conocimiento de problemas ambientales, locales y planetarios.

- Capacitar a personas para analizar, críticamente, la información socio-ambiental.

- Facilitar la comprensión de los procesos ambientales en relación con los sociales, económicos y culturales, de manera política.

- Estimular valores pro-ambientales y fomentar actitudes críticas y constructivas.

- Apoyar el desarrollo de una ética que promueva la protección del ambiente desde una perspectiva de equidad y solidaridad.

- Capacitar a las personas en el análisis de los conflictos socio-ambientales, en el debate de alternativas y en la toma de decisiones para su resolución.

- Fomentar la participación de la sociedad en los asuntos colectivos, potenciando la responsabilidad compartida hacia el entorno.

- Ser instrumento de conductas sustentables en todos los ámbitos de la vida.

La educación ambiental atañe a toda la sociedad, debe tener un enfoque amplio, para potenciar un pensamiento crítico e innovador, que sea capaz de formar una opinión acerca de los problemas socio-ambientales. Con la educación ambiental se pretende fomentar el compromiso de contribuir al cambio social, cultural y económico, a partir del desarrollo de valores, actitudes y habilidades que permitan a toda persona formarse criterios propios, asumir su responsabilidad y desempeñar un papel constructivo (González Gaudiano, 2003).

Una educación ambiental alternativa necesita de una serie de técnicas, métodos, nuevos enfoques teóricos y prácticos, para enfrentar la problemática desde una visión holística de la realidad (ambiente), que permita ver la interconexión de sus dimensiones: culturales, políticas, económicas, sociales, espirituales, legales, éticas y naturales que vive la humanidad, y así contribuir al mejoramiento y desarrollo de la calidad de vida, la recuperación, la conservación y la protección del ambiente. Al final, la educación ambiental debe proponer una alternativa real o soluciones de desarrollo sustentable económica y ecológicamente, justa, mediante cambios estructurales que conlleven a una sociedad acorde con el desarrollo y las necesidades de la humanidad, según sus actuales conocimientos (Guzmán, 2003). La educación ambiental crítica estimula la formación de sociedades socialmente justas y ecológicamente sustentables. Sus principales retos para ser eficiente, pueden apreciarse en la Tabla 2.

Tabla 2

Principales retos de la educación ambiental

$\sqrt{ }$ Consolidarse como una práctica social, ejercida con sus diversas modalidades de educación (formal, informal, no formal y comunitaria).

$\sqrt{ }$ Promover y recuperar valores de conservación, protección y respeto del ambiente.

$\sqrt{ }$ Concienciar a quienes tienen la posibilidad de tomar decisiones que pueden detener o agravar la crisis ambiental.

$\sqrt{ }$ Generar procesos integradores, democráticos y transdisciplinarios para la planificación, ejecución de acciones y su evaluación, tendientes a establecer relaciones armónicas con el ambiente.

$\sqrt{ }$ Abrir espacios a la participación que hace posible que todas(os) sean interlocutoras(es), generadoras(es) e intérpretes de la realidad que nos rodea.

$\sqrt{ }$ Promover la búsqueda de energías y formas de producción limpias.

Nota. Recopilación propia. 


\section{Educación ambiental: características}

El conocimiento educativo oficial, convencional va desde una visión simple lineal acerca del ambiente hasta otra, más compleja del mundo y una ideología basada en la explotación, el dominio, los antagonismos, la competencia y el individualismo; mientras que la educación ambiental supone una visión de mundo compleja e integral. Al respecto, en la Tabla 3 se comparan el enfoque convencional y el enfoque sustentable, para demostrar la especificidad de visión y la relación del mundo.

Tabla 3

Enfoque comparativo

\begin{tabular}{|l|l|}
\hline \multicolumn{1}{|c|}{ Convencional } & \multicolumn{1}{c|}{ Sustentable } \\
\hline Atomista: todo es la suma de las partes. & $\begin{array}{l}\text { Holista: las partes no pueden entenderse fuera de su totalidad, que es } \\
\text { distinta a la suma de sus partes. }\end{array}$ \\
\hline $\begin{array}{l}\text { Mecánico: los sistemas se mueven de un } \\
\text { equilibro a otro. }\end{array}$ & $\begin{array}{l}\text { Sistémico: } \text { interacción de elementos en un contexto y condiciones } \\
\text { dadas, como evolutivo y predecible. }\end{array}$ \\
\hline Universal: existen principios generales. & $\begin{array}{l}\text { Contextualizado: es contingente respecto a un gran número de } \\
\text { factores propios en el tiempo y espacio. }\end{array}$ \\
\hline $\begin{array}{l}\text { Monista: el conocimiento parcial puede } \\
\text { integrarse en un todo. }\end{array}$ & $\begin{array}{l}\text { Subjetivo: no pueden conocerse fuera de nuestras actividades y } \\
\text { valores. }\end{array}$ \\
\hline $\begin{array}{l}\text { Singular: } \text { sólo existe un único elemento o } \\
\text { conocimiento. }\end{array}$ & $\begin{array}{l}\text { Pluralista: } \text { sistemas complejos que se conocen, mediante pautas de } \\
\text { conocimientos alternativas, con singularidades de la realidad. }\end{array}$ \\
\hline $\begin{array}{l}\text { Lineal: el desarrollo avanza sumativo, sin } \\
\text { mirar atrás. }\end{array}$ & $\begin{array}{l}\text { Espiral, } \text { circular: el desarrollo vuelve a su punto de inicio, superando } \\
\text { los obstáculos. }\end{array}$ \\
\hline $\begin{array}{l}\text { Monodisciplinario: fragmenta el } \\
\text { conocimiento de la realidad. }\end{array}$ & $\begin{array}{l}\text { Transdisciplinario: integra aportes diferentes y sistemas de } \\
\text { conocimiento. }\end{array}$ \\
\hline
\end{tabular}

Nota. Tomado de Toledo, 1995.

Hasta ahora, la educación ambiental convencional se ha enfocado con una visión reduccionista, monodisciplinaria, técnica y operativa de la problemática ambiental, sin considerar el alcance y la complejidad que las interacciones humanas tienen en esta situación. La educación ambiental convencional tiene como objeto de estudio la naturaleza no intervenida por el quehacer humano (fotosíntesis, ciclos del agua o del carbono, cadenas alimentarias), lo que es un contrasentido. (Durán, 2002).

¿Cómo hablar del ciclo del carbono sin hacer referencia a la Revolución Industrial y a las emisiones de gases invernadero por parte de las sociedades industrializadas del mundo? ¿Cómo hablar de la destrucción de las selvas tropicales sin explicar los fenómenos de la pobreza y la injusta distribución de la riqueza en el mundo? ¿Cómo hablar de deforestación sin obviar la voracidad de las transnacionales y los agroexportadores locales?

La educación ambiental debe ser un proceso que genere aprendizajes mediante la construcción y reconstrucción de conocimientos, como resultado del estudio de las complejas interacciones sociedad-ambiente, lo que ha de generar conciencia en la ciudadanía de su papel como parte integrante de la naturaleza, para que desarrollen nuevas relaciones, sentires, actitudes, conductas y comportamientos hacia ella. Pero, también, debe llevarnos a reflexionar, a cuestionar y a valorar los estilos de desarrollo y de progreso convencional, que se basan en la degradación de las aguas, de la tierra, del aire y no resuelve los problemas sociales (pobreza, miseria, concentración de riquezas, violencia y otras) (Martínez, 2007b). 
Los programas de educación ambiental deben ser cuidadosamente dirigidos hacia las necesidades específicas locales de los destinatarios. La población debe ser educada en los fenómenos y problemas ambientales locales, nacionales y globales y, para ello, se utilizan los métodos y las estrategias de la educación formal.

Un programa de educación ambiental debe tener en cuenta el ambiente en su totalidad: natural y cultural; social, político, económico, tecnológico, social, legislativo, y estético. Debe hacer hincapié en una participación integral y activa, en la prevención y resolución de los problemas concretos y generales. Además, debe considerar todo desarrollo y crecimiento en una perspectiva ambiental; pedagógicamente, fomentar el valor y la necesidad de la cooperación local, nacional e internacional (Meza Aguilar, 1992). Eso implica un enfoque que trasciende lo biológico, para ampliar el concepto de ambiente al considerar, también, las relaciones económicas, políticas, socio-culturales y naturales.

\section{Aspectos políticos de la educación ambiental}

El proceso de aprendizaje no puede ser neutral ante la situación actual de crisis social y ambiental. Ésta debe ser crítica ante las políticas de desarrollo económico-productivo de corte neoliberal y su énfasis en el mercado desregularizado y sin control, pues aunque maximiza la producción y genera gran riqueza, ésta no se refleja en una justa distribución social, sino que se privatiza y no aporta en la resolución del problema ambiental; todo lo contrario, lo profundiza al deteriorar el ciclo de regeneración del gran ecosistema, la biosfera del planeta Tierra (Delgado, 2006).

Así pues, la educación en lo social y ambiental no es neutral (Freire, 1995), sino que es un acto político, sirve para la libertad y el desarrollo del ser humano y la sociedad; por eso, debe ocuparse de la dinámica del ambiente físico-biológico, del socio-económico y del desarrollo humano, tanto material, como espiritual, e integrarse en todas las disciplinas. Se debe forjar un proceso de educación comprometida, para cambiar y plantear una nueva forma de vida y de mentalidad. Ello requiere de la participación democrática y de la distribución más justa del poder político, económico, cultural, social en función de la protección de lo natural.

La problemática socio-ambiental no es neutral ni ajena a los intereses políticos y económicos que actualmente rigen el mundo. Para que ésta sea válida y efectiva, tiene que cuestionar esa supuesta neutralidad axiológica de la crisis. He aquí la importancia de las ciencias sociales y naturales en la educación ambiental.

Para proponer algunas soluciones, dadas en el asunto de la politicidad de la educación, Marx plantea el carácter político-ideológico de los contenidos en la educación. Pero, ¿cuál es la esencia de lo político en educación? Freire (1995) realiza un importante aporte, al orientar el análisis a las formas que adquiere la transmisión de saberes. Incorporando su carácter ideológico, capta otro aspecto político presente en la práctica educativa: el tipo de vínculo entre el que educa, el que aprende y el de ambos con el conocimiento y la realidad.

La educación ambiental ha de caracterizar los principales cambios estructurales de la sociedad y su correlación con la realidad política, económica y cultural, enmarcada en un análisis de causa-efecto, cronología y aspectos de la crisis socio-ambiental en los ámbitos mundial, nacional, regional, que tome en cuenta su manifestación en nuestra realidad inmediata: hogar, comunidad, centro educativo, lugar de trabajo, ciudad, barrio, caserío, distrito o cantón, otros espacios de convivencia. Por eso, no se pueden obviar las situaciones polémicas que se generan en los niveles nacional e internacional, las que, muchas veces, son impuestas y generan la alteración ambiental: como son 
los modelos de desarrollo (agroexportación, sustitución de importaciones, los ajustes y, finalmente, el TLC-ALCA), impulsados por los países industrializados, aceptados y aplicados por las élites dominantes, cómplices internas de los países en vías de desarrollo.

En los programas de educación ambiental, el contenido se orienta hacia el estilo de vida y las relaciones sociedad-naturaleza, hacia los problemas concretos e inmediatos.

Un programa crítico de educación ambiental forja una conciencia, la actitud y las competencias ambientales necesarias para proteger el planeta y toma en cuenta el tipo de relaciones que la sociedad establece entre sí y la naturaleza, con sus causas y efectos; además de vincular al educando con la comunidad, mediante el estímulo de los valores y las actitudes que promueven un comportamiento dirigido hacia la transformación racional de la realidad, en sus aspectos naturales y sociales, y desarrolla, en el educando, las habilidades y las aptitudes necesarias para dicha transformación (Sessano, 2006).

\section{Transversalidad y transdisciplinariedad: concepto y características}

La transdisciplinariedad es "uno de los más altos ideales de la educación ambiental, en que, los objetivos ambientales pueden sólo ser logrados tratando los temas a través de todas las áreas y formando nuevas asociaciones" (Schrodinger, 1998, p. 38). Sus características más sobresalientes son:

a- Enfoque holístico, sistémico, entrópico, dialéctico del ambiente.

b- Enfoque transdisciplinario e interacción.

c- Resolución de problemas.

d- Metodología participativa y equitativa.

e- Reconocimiento de la biodiversidad y cultural.

f- Solidaridad, tolerancia.

g- Pensamiento constructivo e innovador.

h- Enfoque interpretativo, cualitativo.

La transversalidad hace referencia a la diversidad de los enfoques transdisciplinarios con los que puede ser tratado un mismo tema de interés general y particular de relevancia ambiental, social y otros (Gallo, 1999). Asimismo, involucra el sobrepaso de los límites entre distintas disciplinas, que puede contribuir al desarrollo de nuevos conceptos y realidades.

Un tema transversal concreto de la educación ambiental, son los contenidos educativos que forman parte de las áreas del currículo; los cuales se refieren a actitudes y valores, hacia la vida cotidiana, la realidad y la problemática socio-ambiental. Esta opción se inscribe dentro de la concepción constructivista del proceso educativo, al permitir al sujeto en situación de aprendizaje, la construcción de un conocimiento que implica un significado real en su vida. El abordaje de temas transversales debe efectuarse en los niveles de complejidad y estrategias apropiados, considerando los conocimientos adquiridos, los intereses y posibilidades de las y los estudiantes.

\section{Estrategias para el desarrollo de la educación ambiental}

La sociedad (administradores de recursos naturales, científicos, políticos y público, en general) debe elaborar estrategias para un adecuado manejo de los bienes (tierra, agua, otros), que sean sustentables en los campos económico, político, social y cultural. 
Entre las estrategias claves para el desarrollo de éstas están:

a. Estrategia múltiple. La educación ambiental tiene un carácter integrador y globalizador, para desarrollar una nueva visión del mundo, más social, más sensible a lo ambiental, con un sentido entrópico, sistémico, es decir, holístico. Al ser transdisciplinaria, debe ser aplicada desde varias áreas disciplinarias en forma conjunta: perspectiva científica (ecología, biología, sociología, economía, política), cultural (ideología, valores, conductas, actitudes, tradiciones, espiritualidad) e integradoras (aprendizaje, recreación).

El aporte se da en dos perspectivas teóricas: constructivista y sistémica, en los procesos de aprendizaje y aplicación de la educación ambiental, de forma gradual y progresiva, que genere la reconstrucción de un punto de referencia didáctico e integre la reflexión psicológica, epistemológica y socio-política, en un marco teórico (Martínez, 2005).

Este enfoque curricular merece una nueva perspectiva, pues es necesaria la aplicación diversa del enfoque transdisciplinario. Estos temas reflejan aspectos sociales, políticos, económicos y culturales, es decir, el estilo de desarrollo, sus características, desde un ángulo de causa-consecuencia, su impacto socio-ambiental y posibles alternativas metodológicas de analizar y de plantear soluciones.

b. Investigación de situaciones problemáticas. La metodología permite abordar el estudio de problemas socio-ambientales con potencialidad integradora para trabajar contenidos científicos y cotidianos, en el proceso de aprendizaje. El proceso de abordar situaciones problemáticas, contribuye a que los seres humanos construyan nuevos conocimientos, de tal forma que aprendan en la medida que trabajan con esas problemáticas y elaboran respuestas (cognitivas, afectivas, conductuales) (Martínez, 2005).

En lugar del conocimiento educativo, podría darse la propuesta de problemáticas educativas, entendida como problemas que van formulando planteamientos sencillos a más complejos y hacia verdaderas problemáticas de carácter socio-ambiental. La investigación del ambiente, por parte de las y los estudiantes, debe integrarse al currículo, enfocada como una aproximación a la temática ambiental, que se realiza en los centros educativos, y modificar la dinámica de los procesos de aprendizaje.

c. Aprendizaje significativo. Se caracteriza por implicar que el alumno comprenda conceptos, procedimientos, actitudes y valores y no sólo los memorice. Con el aprendizaje significativo, los nuevos conocimientos se incorporan en la estructura cognitiva de modo no memorístico ni mecánico. Se supone que quien aprende dispone, necesariamente, de ciertos conocimientos, conceptos, ideas y esquemas. Éstos son acumulados durante experiencias previas, aunque sencillas y sirven como "ideas inclusoras", referentes u orientadores para interpretar, asociar y dar sentido a los nuevos conocimientos que se van adquiriendo. De lo contrario, es poco probable que logre su comprensión. Así, "la idea inicial para promover el aprendizaje significativo sería... tener en cuenta los conocimientos factuales y conceptuales que el alumno ya posee -así como sus actitudes y procedimientos- y cómo van a interactuar con la nueva información proporcionada por los materiales de aprendizaje" (Pozo,1995, p 36).

d. Actitud en la educación ambiental. En su construcción, los estudiantes deben pasar de una concepción analítica del ambiente (realidad: una suma de las partes) a una visión sistémica (realidad: una jerarquía de sistemas integrados unos con otros). Paralelamente, debe sustituirse el enfoque descriptivo de la realidad, en la que no se plantea la relación causaefecto de las cosas, por el análisis de explicaciones causales, primero lineales (un factor del ambiente determina a otro), pasando por interactivas (los dos factores se determinan 
mutuamente) y, luego, espirales (visto como un proceso vivo), para llegar a la holística (todo se interactúa e integra de alguna manera) (Sarabia, 1994).

Esta progresión transciende las relaciones simples (un animal se come a otro) al reconocimiento de relaciones complejas (redes tróficas, ciclos), así como la superación de una visión del mundo egocéntrica (todo está en función de uno mismo), antropocéntrica (realidad vista desde la óptica de lo humano) o bien, mercadocéntrica (realidad valorada desde el mercado) y sociocéntrica (los hechos se analizan desde la perspectiva socio-cultural del sujeto), ignorándose otras posibles perspectivas culturales (mediante una concepción relativa), en la que el individuo o grupo podría adoptar distintas perspectivas y considerar diversos aspectos de la realidad o el problema, hasta alcanzar la biocéntrica (el centro de todo es la biosfera) o ecocéntrica (el centro es toda la naturaleza) (Sessano, 2006).

\section{Ética y educación ambiental}

La "crisis ambiental" se ha generado por la forma en que el ser humano o la sociedad, en general, se han relacionado con el ambiente (apropiación, producción y consumo). La responsabilidad de todos es la ubicación epistemológica de quienes deben conocer las relaciones sustentables con el ambiente y la sociedad.

La crisis ambiental es un problema de responsabilidad ante la vida, ante la historia y ante sí, consiste en que la transformación de la naturaleza tiene como centro los intereses privados, pone en peligro la producción y la reproducción de la vida humana y de todos los seres vivos que dependen del ambiente.

En la orientación hacia valores, la ética ambiental se basa en el principio de solidaridad al concebir al planeta como el espacio geográfico donde los seres humanos debieran compartir y disfrutar sus bienes, mediante el manejo sustentable de los bienes naturales. Uno de los aspectos que identifican a la educación ambiental es su orientación hacia los valores, o la inclusión de una ética de las relaciones entre el ser humano y su ambiente, y la consideración de éste como un bien por preservar.

Según Marina (1995), algunos aspectos éticos que pueden considerarse en una experiencia de aprendizaje-servicio en la educación ambiental, son los siguientes

- Interés de las personas afectadas por los problemas socio-ambientales.

- Interés de la sociedad, amenazada en su calidad de vida y degradación futura.

- Reconocimiento del derecho a la vida y a la calidad de vida de la población.

- Derecho a la vida de otras especies amenazadas por el ser humano.

La educación ambiental debe propiciar un cambio de actitudes, una participación responsable en la gestión social del ambiente y crear acciones adecuadas con su entorno, en general. Pero la educación convencional ha sido poco eficaz con respecto al cambio de actitudes y de comportamientos socio-ambientales. Por eso, se debe redimensionar la educación ambiental y encontrar rasgos que la identifiquen e impulsen hacia una acción formativa, dirigida al cambio actitudinal o de comportamientos de un colectivo dado.

Entre los objetivos ambientales de la educación ambiental, para interrelacionar lo actitudinal con lo conceptual, aplicada en el sistema educativo, de acuerdo con Caduto (1992) y con Puig Rovira (1992), están:

$\sqrt{ }$ Desarrollar la concienciación ante situaciones socio-ambientales, y mejorar la capacidad de 
plantearse problemas, debatirlos, construir opiniones propias, difundir esas problemáticas y sus propuestas de solución.

$\sqrt{ }$ Promover la sensibilización de las propias actitudes y comportamientos habituales, en el entorno, mediante la valoración de las repercusiones de impactos importantes.

$\sqrt{ }$ Estimular la comprensión compleja de los conceptos básicos y el funcionamiento del ambiente, a causa de la constante interacción, cambio y evolución.

$\sqrt{ }$ Generar actitudes de solidaridad, mediante las interconexiones existentes entre el ambiente y la interdependencia socio-económica, política y cultural del mundo, y superar la separación rígida: la dimensión natural y social del ambiente.

$\sqrt{ }$ Valorar el patrimonio cultural de la propia sociedad y la naturaleza en que se vive, como parte del respeto hacia la diversidad cultural y ecológica.

$\sqrt{ }$ Desarrollar un conocimiento de la dinámica del entorno, la actitud y capacidad de disfrutar el ambiente, armónicamente, entre el uso individual y colectivo.

$\sqrt{ }$ Mejorar la capacidad de análisis, actitudes y comportamientos en la vida cotidiana, y ambientar las acciones personales diarias y su integración en la sociedad, desde una perspectiva planetaria de una ética ambientalista.

\section{Conclusión}

La necesidad de organizar la economía según la justicia, el respeto al ser humano y a los bienes naturales, ya no es una cuestión de opinión moral o política, sino un imperativo que nos exige poner de nuestra parte. El consumidor es el último eslabón del sistema económico y, como tal, tiene la responsabilidad y el poder de cambiar el estado a que se nos induce por los medios de comunicación social (Teitelbaum, 2002).

El desarrollo insustentable no es resultado de una mala planificación o ejecución de un proyecto, ni falta de visión o mala estructuración institucional, ni temporal. El modelo agroindustrial se agota, pues no es la solución, como pretenden los gobiernos (de político-empresarios), sino que es la causa de la crisis socio-ambiental actual. Tampoco es un problema técnico, ni tecnológico, ni económico, ni social; es ético, es moral, es político, con sus estructuras de relaciones deshumanizadas y desnaturalizadas, que enfatizan en mercados desregularizados. El desarrollo dominante no comparte de modo equitativo, ni toma en cuenta las necesidades locales para eliminar la pobreza y el hambre, debidamente. Éste enfatiza en maximizar la producción y el consumo para resolver sus intereses externos, en detrimento de las necesidades y las responsabilidades socio-ambientales locales (Martínez, 2007b). Por eso, se debe replantear todo el sistema de dominación convencional (instituciones, leyes), que, como modelo, está en franca decadencia e implantar uno que rompa con las estructuras locales, nacionales e internacionales, más acorde con la realidad y la necesidad de los tiempos.

Ello implica, asumir el mensaje de la conciencia lógica humana, bajo un manejo sustentable con la vida natural. Representa todo un cambio en nuestra forma de conocimiento y cosmovisión; pero, también, en nuestra ética y estilo de vida dominante. Por ello, la educación ambiental se constituye en el elemento básico para enfrentar la crisis del entorno y mejorar la calidad de vida de los seres humanos.

Hoy, la educación ambiental crítica trabaja, intensamente, en la generación de nuevos conocimientos, metodologías, técnicas y estimulo de nuevos valores sociales y culturales, orientada a que todas las disciplinas contribuyan a un desarrollo sustentable.

En la medida en que las educadoras y los educadores socio-ambientales interpreten la 
interacción (histórica) del conocimiento, creencia y ambiente, apliquen la educación de manera holística y analicen, críticamente, los problemas, los aciertos y las necesidades actuales, en esa medida estarán proponiendo planes de manejo sustentable de los ecosistemas, que aseguren la participación de la población a una existencia digna. Sin embargo, para generar un proceso de políticas socio-ambientales es necesario cuestionar el desarrollo convencional con cambios estructurales (institucionales, legislativos), en los que el ciclo económico respete el ciclo ecológico. Estos cambios deben implicar una relación socio-económica y ambiental más justa y equitativa que beneficie a la mayoría.

Se trata de tomar partido por una opción viable para el futuro de la humanidad y la naturaleza. La concepción sustentable y su relación con su entorno son, fundamentalmente, una opción de vida, una opción humana, que sólo es proporcionada, realmente, por la educación ambiental, como alternativa de desarrollo integral. Por eso, tener conciencia humana es tener conciencia política.

\section{Referencias bibliográficas}

Álvarez, A. (comp.). (2003). Memorias del III Congreso Iberoamericano de Educación Ambiental. MARN / Caracas, Venezuela: Fundación Polar.

Beck, U. (2004). Poder y contrapoder en la era global: la nueva economía política mundial. Barcelona, España: Paidós.

Bedoy, V. (2000). La historia de la educación ambiental: reflexiones pedagógicas. En Educar. REVISTA DE EDUCACIÓN / NUEVA ÉPOCA NÚM. 13/ABRIL-JUNIO 2000. (Versión electrónica, disponible en http://educacion.jalisco.gob.mx/consulta/educar/13/13Bedoy.html)

Caduto, M. (1992). Guía para la enseñanza de valores ambientales. Programa Internacional de Educación Ambiental. Madrid, España: UNESCO-PNUMA.

Caride, J. A. (2000). Educación ambiental y desarrollo humano: Nuevas perspectivas conceptuales y estratégicas. Conferencia dictada en el III Congreso Iberoamericano de Educación Ambiental. Caracas, Venezuela.

Delgado, C. J. (2006). La educación ambiental desde la perspectiva política. En Cuba Verde. La Habana, Cuba: Editorial Félix Varela.

Durán, D. (2002). Escuela, ambiente y comunidad. Manual de capacitación docente. Integración del aprendizaje-servicio y la educación ambiental. Fundación Educambiente. Buenos Aires, Argentina: Programa Escuelas Solidarias.

Febres-Cordero, M. E. \& Florián, D. (2002). Políticas de educación ambiental y formación de capacidades para el desarrollo sustentable. De Río a Johannesburgo. La transición hacia el desarrollo sustentable. Seminario organizado por el PNUMA/INE-SEMARNAT/ Universidad Autónoma Metropolitana.

Fien, J., Scott, W. \& Tilbury, D. (1999). Education and Conservation: An Evaluation of the 
Contribution of Educational Programmes to Conservation within the WWF Network: Final Report to World Wildlife Fund. Washington, D. C.

Foladori, G. \& González Gaudiano, E. (2003). En pos de la historia en educación ambiental. Revista Tópicos de Educación ambiental, 3(8), 28-43.

Freire, P. (1995). La educación como una acción cultural. San José, Costa Rica: EUNED.

Gallo, S. (1999). Conocimiento y transversalidad. Universidad Metodista de Piracicaba. Recuperado el 12 de marzo de 2001, de www.bu.edu/wcp/papers/Tkno/TknoGall.htm

Guzmán, A. (Ago., 2003). Educación socio-ambiental y problemática medio-ambiental global. Ecoportal. Versión electrónica, disponible en www.eco-portal.net

Hernández, A., Ferriz, Á., Herrero, Y., González, L., Morán, C., Brasero, A. et al. (2010). La crisis ecosocial en clave educativa. Guía didáctica para una nueva cultura de paz. Madrid, España: Centro de Investigación para la Paz (CIP-Ecosocial).

Labrador, C. \& del Valle, Á. (1995). La educación medioambiental en los documentos internacionales. Notas para un estudio comparado. Revista Complutense de Educación. Vol. 6(2), 75-94. (Versión electrónica, disponible en http://revistas.ucm.es/edu/11302496/articulos/ RCED9595220075A.PDF)

Marina, J. A. (1995). Ética para náufragos (4ª ed.). Barcelona, España: Editorial Anagrama.

Martínez, R. (1998). Reflexión sobre educación, sociedad y ambiente. Revista Tópicos, 41, s. p.

Martínez, R. (2005). Fundamentos culturales, sociales y económicos de la agroecología. Revista Ciencias Sociales, № 13-14, s. p.

Martínez, R. (2007b). Aspectos políticos de la educación ambiental. Revista Electrónica Actualidades Investigativas en Educación (INIE), Vol. 7(3), 1-25.

Meza Aguilar, L. (Nov.-Dic., 1992). Educación Ambiental... ¿para qué? Nueva Sociedad, 122, 176-185.

Pozo, J. (1995). El aprendizaje y la enseñanza de hechos y conceptos. En Coll, C., Pozo, J. I., Sarabia, B. y Valls, E. Los contenidos en la Reforma. Enseñanza y aprendizaje de conceptos, procedimientos y actitudes. (pp. 19-79). Madrid, España: Santillana.

Puig Rovira, J. M. (1992). Educación moral y cívica, una de las guías para los temas transversales. Madrid, España: Materiales de la Reforma.

Sarabia, B. (1994). El aprendizaje y la enseñanza de hechos y conceptos. En Coll, C., Pozo, J. I., Sarabia, B. y Valls, E. Los contenidos en la Reforma. Enseñanza y aprendizaje de conceptos, procedimientos y actitudes. (s. pp.). Madrid, España: Santillana.

Schrodinger, E. (1998) (Trad. Jaime Fingerhut y Arthur Klein). Mi concepción del mundo seguido 
de mi vida. (2 ed.). (Serie Metatemas). Barcelona, España: Tusquets.

Sessano, P. (Abr., 2006). La educación ambiental: un modo de aprender. Anales de la educación común. Tercer siglo. Año 2. $\mathrm{N}^{\mathrm{o}} 3$, s. p.

Teitelbaum, A. (2002). El papel de la educación ambiental en América Latina. París, Francia: UNESCO.

Toledo, V. M. (1995). Campesinidad, agroindustrialidad, sostenibilidad: los fundamentos ecológicos e históricos del desarrollo rural. Cuadernos de Trabajo 3:1-45, Grupo Interamericano para el Desarrollo Sostenible de la Agricultura y de los Recursos Naturales. México.

UNESCO. (2004). Education for a Sustainable Development. Recuperado el 18 de enero de 2005, de http://portal.unesco.org.education/en/ev.php-URL ID=23298\&URL_DO=DO TOPIC\&URLSECTION=201html

Wood, D. \& Walton, D. (1990). Cómo planificar un programa de educación ambiental. (Cuadernos de la FAO). Washington D. C., USA: IIED y USFWS.

\section{Otras fuentes consultadas}

Díaz-Aguado, M. J. \& Medrano, C. (1995). Educación y razonamiento moral. Una aproximación constructivista para trabajar los contenidos transversales. Bilbao, España: Editorial Mensajeras.

Leff, E. (1997). Conocimiento y educación ambiental. Formación Ambiental, 7(17), 19-22.

Martínez, R. (2007a, Junio). Educación ambiental y sustentabilidad. Centro Cultural Poveda, Anuario Pedagógico, 10, s. p.

UNESCO. (1980). La educación ambiental. Las grandes orientaciones de la conferencia de Tbilisi (1977). París, Francia: Autor. 